\title{
Genetic Identification of Spirometra decipiens Plerocercoids in Terrestrial Snakes from Korea and China
}

\author{
Hyeong-Kyu Jeon ${ }^{1}$, Hansol Park', Dongmin Lee', Seongjun Choe', Kyu-Heon Kim², Woon- Mok Sohn³, \\ Keeseon S. Eom ${ }^{1, *}$ \\ ${ }^{1}$ Department of Parasitology, Medical Research Institute and Parasite Resource Bank, Chungbuk National University School of Medicine, Cheongju \\ 28644, Korea; ' 2 Ministry of Food and Drug Safety, Cheongju 28159, Korea; ${ }^{3}$ Department of Parasitology and Tropical Medicine, Institute of Health \\ Sciences, Gyeongsang National University College of Medicine, Jinju 52727, Korea
}

\begin{abstract}
Human sparganosis is a zoonotic disease caused by infection with larval forms (procercoid/plerocercoid) of Spirometra spp. The purpose of this study was to identify Spirometra spp. of infected snakes using a multiplex PCR assay and phylogenetic analysis of mitochondrial DNA sequence data from the spargana of terrestrial snakes obtained from Korea and China. A total of 283 snakes were obtained that included 4 species of Colubridae comprising Rhabdophis tigrinus tigrinus $(\mathrm{n}=150)$, Dinodon rufozonatum rufozonatum $(\mathrm{n}=64)$, Elaphe davidi $(\mathrm{n}=2)$, and Elaphe schrenkii $(\mathrm{n}=7)$, and 1 species of Viperidae, Agkistrodon saxatilis $(n=60)$. The snakes were collected from the provinces of Chungbuk, Chungnam, and Gyeongbuk in Korea $(n=161)$, and from China $(n=122)$. The overall infection rate with spargana was $83 \%$ (235/283). The highest was recorded for $D$. rufozonatum rufozonatum (100\%), followed by $A$. saxatilis (85\%) and $R$. tigrinus tigrinus (80\%), with a negative result for $E$. davidi $(0 \%)$ and $E$. schrenkii $(0 \%)$. The sequence identities between the spargana from snakes $(n=50)$ and Spirometra erinaceieuropaei (KJ599680) or S. decipiens (KJ599679) control specimens were $90.8 \%$ and $99.2 \%$, respectively. Pairwise genetic distances between spargana $(n=50)$ and $S$. decipiens ranged from 0.0080 to 0.0107 , while those between spargana and S. erinaceieuropaei ranged from 0.1070 to 0.1096 . In this study, all of the 904 spargana analyzed were identified as $S$. decipiens either by a multiplex PCR assay $(n=854)$ or mitochondrial cox1 sequence analysis $(n=50)$.
\end{abstract}

Key words: Spirometra decipiens, sparganum, plerocercoid, terrestrial snake, mitochondrial cox1 gene, Korea, China

\section{INTRODUCTION}

Human sparganosis is a zoonotic disease caused by infection with the larval forms (procercoid/plerocercoid) of Spirometra spp. The first recorded case of human sparganosis in Korea involved a farmer [1] based on a report by Uemura in 1917. Cho et al. [2] reviewed 63 cases of indigenous sparganosis in 1975 in Korea. Then, an additional 56 cases were presented during the period from 1975 to 1989 [3]. The most recent report published in 2015 details human sparganosis cases recorded for the period 1979 and 2009 in Korea involved Spirometra erinaceieuropaei (Rudolphi, 1819) ( $\mathrm{n}=35)$ and Spirometra decipiens (Diesing, 1850) $(\mathrm{n}=15)[4]$. As a consequence, S. decipiens was added to the causative agent list of human sparganosis that

\footnotetext{
- Received 10 January 2016, revised 15 March 2016, accepted 20 March 2016.

*Corresponding author (kseom@chungbuk.ac.kr)

(c) 2016, Korean Society for Parasitology and Tropical Medicine

This is an Open Access article distributed under the terms of the Creative Commons Attribution Non-Commercial License (http://creativecommons.org/licenses/by-nc/3.0) which permits unrestricted non-commercial use, distribution, and reproduction in any medium, provided the original work is properly cited.
}

was previously regarded as only involving S. erinaceieuropaei.

The routes of spargana infection involve either drinking water contaminated with procercoid-infected copepods or consumption of undercooked meat of plerocercoid-infected snakes or frogs. Additionally, infection can occur by placement of poultices that use the skin of infected snakes or frogs. Snakes have been noted as a very important source of infection for human sparganosis by many researchers in Korea [57]. At least 7 species of terrestrial snakes have thus far been investigated as possible sources of infections with spargana, among which the following 6 species of snakes have been positively identified with spargana: Elaphe rufodorsata, Elaphe dione, Dinodon rufozonatum ruforzonatum, Rhabdophis tigrinus tigrinus, Zamenis spinalis, and Agkistrodon halys. All of these snakes were from Gangwon-do (Province), which is located at the eastern side of the peninsula [8]. Spargana have been found in various species of frogs, snakes, birds, and mammals in the Far East region, including China and Japan, and 6 spirometrid species (under the name Diphyllobothrium) were reported with morphological and biological studies on the species in China [9]. 
The present study was to provide information for what Spirometra species are being distributed in Korea and China by using genetic analysis.

The morphological identification of spargana is usually difficult because they lack distinguishing characteristics. More recently, molecular approaches to differential identification of $S$. erinaceieuropaei and S. decipiens have been introduced that analyze sequence differences of mitochondrial genomes $[4,10]$. In the present study, we used species-specific primers for a multiplex PCR assay based on mitochondrial sequences of $S$. erinaceieuropaei and S. decipiens. The larval forms of Spirometra spp. (spargana) collected from terrestrial snakes in Korea and China were identified by the multiplex PCR assay and through phylogenetic analysis of mitochondrial DNA sequence data.

\section{MATERIALS AND METHODS}

\section{Snakes}

A total of 283 snakes were obtained from the provinces of Chungbuk, Chungnam, and Gyeongbuk in Korea and from unknown localities in China during the period from 1993 to 2001. Among these specimens, 161 Korean snakes (1993-2002) were donated by Association of Wild Animals Protection for research purpose and included $R$. tigrinus tigrinus $(\mathrm{n}=150), D$. rufozonatum rufozonatum $(\mathrm{n}=4)$, Elaphe davidi $(\mathrm{n}=2)$, Elaphe schrenckii $(\mathrm{n}=2)$, and Agkistrodon saxatilis $(\mathrm{n}=3)$. The remaining 122 Chinese snakes had been smuggled from China and subsequently donated to Chungbuk National University School of Medicine by the Busan Customs of Korea in 2002 for research purpose and included $D$. rufozonatum rufozonatum $(\mathrm{n}=60)$, E. schrenckii $(\mathrm{n}=5)$, and A. saxatilis $(\mathrm{n}=57)($ Table 1$)$.

\section{Nucleotide sequencing and multiplex PCR}

A total of 904 plerocercoids were subjected to molecular biological analysis in this study. This included 50 plerocercoids for the analysis of mitochondrial cox 1 gene sequences comprising 10 plerocercoids from each of 5 snake species. The re- maining 854 plerocercoids were analyzed using a multiplex PCR assay. A single plerocercoid larva was chopped into small pieces on a glass slide, and total genomic DNA was extracted with a DNeasy tissue kit (Qiagen, Valencia, California, USA) according to the manufacturer's instructions. PCR and DNA sequencing were performed according to protocols previously established [4].

For multiplex PCR, the forward primer, Se/Sd-7963F (5'-ACG TGG TTT GTG GTG GCT CAT TTT-3', nucleotides 7963-7986 from specimen numbers KJ599679 and KJ599680 uploaded to GenBank) was designed from the highly conserved sequences of the S. decipiens and S. erinaceieuropaei cox 1 gene. The first reverse primer Se-8344R, specific for S. erinaceieuropaei (5'-TGG TCA CCT ATA CCC TAT CAT-3', nucleotides 8344-8364 from KJ599680) was designed to amplify different sized products from the second one. The second reverse primer was Sd8584R, which was specific for $S$. decipiens (5'-GTA TCA AGT TGG TTA GGA AGT TAA-3', nucleotides 8584-8607 from KJ599679). In order to test differential diagnosis of spirometrid tapeworms, multiplex PCR was performed using 15 DNA samples that comprised $S$. erinaceieuroapei plerocercoids $(\mathrm{n}=8)$ from humans and $S$. decipiens plerocercoids $(\mathrm{n}=7)$ from snakes. These DNA samples were confirmed by analysis of cox1 gene sequences.

\section{DNA sequence analyses}

DNA sequences were assembled and aligned with Geneious 6.1.5 (Biomatter, Auckland, New Zealand). The sequenced regions were identified by comparison with sequences of $S$. decipiens (KJ599679) and S. erinaceieuropaei (KJ599680), which were available in GenBank database. The phylogenetic analyses were performed with PAUP 4.0 [11] using the neighborjoining (NJ), maximum parsimony (MP), and maximum likelihood (ML) methods, with D. nihonkaiense and D. latum as out-groups. Phylogenetic trees were constructed using the partial mitochondrial cox1 sequences ( $374 \mathrm{bp}$ ) of 5 taxa of Diphyllobothriidae as represented by $S$. decipiens (KI599679), $S$.

Table 1. Infection rate of spargana in snakes collected between 1993 and 2002

\begin{tabular}{lcccc}
\hline Species of snake & No. examined & No. of snakes with spargana (\%) & Total no. collected & Av. no. per snake \\
\hline Elaphe schrenkii & 7 & 0 & $(0.0)$ & 0 \\
Elaphe davidi & 2 & 0 & $(0.0)$ & 0 \\
Dinodon ruforzonatum ruforzonatum & 64 & $64(100.0)$ & 114 \\
Rhabdophis tigrinus tigrinus & 150 & $120(80.0)$ & 360 \\
Agkistrodon saxatilis & 60 & $51(85.0)$ & 430 \\
Total & 283 & $235(83.0)$ & 3.0 \\
\hline
\end{tabular}


erinaceieuropaei (KJ599680), S. proliferum (AB015753), D. nihonkaiense (EF420138), and D. latum (DQ985706). Pairwise genetic distances based on the partial cox1 sequences (374 bp) were analyzed using the NJ method with the K2P substitution model. The parasite materials used in this study were provided by the Parasite Resource Bank of Korea, National Research Resource Center, the Republic of Korea.

\section{RESULTS}

\section{Sparganum infection in snakes}

The 283 snakes subjected to this study were identified as 1 of the 4 species of Colubridae; $R$. tigrinus tigrinus $(\mathrm{n}=150), D . r u$ fozonatum rufozonatum $(\mathrm{n}=64)$, E. davidi $(\mathrm{n}=2)$, and E. schrenkii $(n=7)$, or a species of Viperidae; A. saxatilis $(n=60)$. With the exception of $E$. schrenkii and $E$. davidi, spargana were found in specimens of the remaining 3 species of snakes. The overall prevalence of spargana was $83 \%$ (235/283), with the highest infection rate being recorded for D. rufozonatum rufozonatum (100\%), followed by A. saxatilis (85\%) and R. tigrinus tigrinus $(80 \%)$ (Table 1). A total of 904 plerocercoids were collected from these snakes. The average number of spargana per infected snake ranged 1.8-8.4, depending on the snake species.

\section{Sequence divergence of mitochondrial cox1}

The difference in nucleotide sequences of the partial cox1 gene between S. erinaceieuropaei (KJ599680) and S. decipiens (KJ599679) was 9.2\%. Analysis of spargana specimens collected from snakes $(n=50)$ revealed sequence identities with $S$. erinaceieuropaei and S. decipiens of $90.8 \%$ and $99.2 \%$, respectively. The pairwise genetic distance between our spargana specimens $(\mathrm{n}=50)$ and $S$. decipiens ranged 0.0080-0.0107 while that with S. erinaceieuropaei ranged 0.1070-0.1096 (Table 2). Based on the phylogenetic relationships between the spargana, the Diphyllobothrium spp. were shown to be basal to the Spirometra spp. clades, as determined using the different analytical meth-

Table 2. Percentage sequence differences of the mitochondrial cox1 gene between spargana isolated from various snakes and Spirometra sp., Diphyllobothrium latum, and D. nihonkaiense

\begin{tabular}{lccccc}
\hline Species Larval stage & S. decipiens & S. erinaceieuropaei & S. proliferum & D. nihonkaiense & D. latum \\
\hline Plerocercoid $^{(}(n=10)$ & 0.0080 & 0.1070 & 0.1070 & 0.1551 & 0.1604 \\
Plerocercoid $(n=10)^{\text {Plerocercoidc }(n=10)}$ & 0.0107 & 0.1070 & 0.1070 & 0.1631 & 0.1684 \\
Plerocercoid $^{d}(n=10)$ & 0.0080 & 0.1070 & 0.1070 & 0.1551 & 0.1604 \\
Plerocercoide $(n=10)$ & 0.0107 & 0.1096 & 0.1070 & 0.1578 & 0.1631 \\
\hline
\end{tabular}

aPlerocercoids from Dinodon ruforzonatum ruforzonatum in Korea.

${ }^{b}$ Plerocercoids from $D$. ruforzonatum ruforzonatum in China.

'Plerocercoids from Rhabdophis tigrinus tigrinus in Korea.

dPlerocercoids from Agkistrodon saxatilis in Korea.

ePlerocercoids from $A$. saxatilis in China.

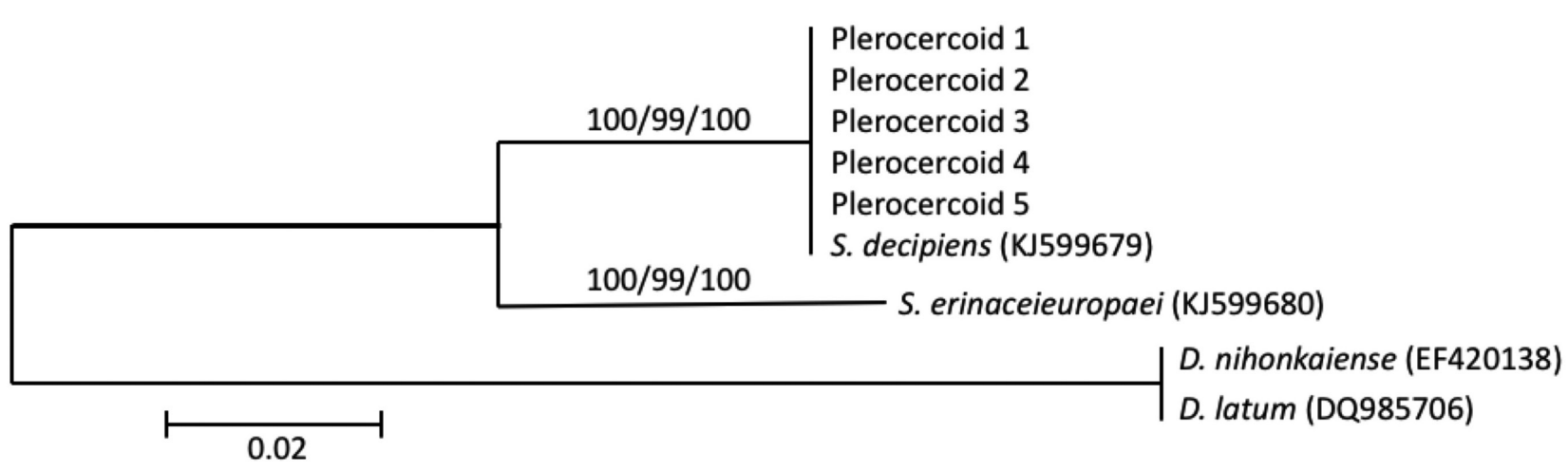

Fig. 1. A phylogenetic tree inferred from mitochondrial cox1 sequence data from Spirometra decipiens and related Diphyllobothrium species by the neighbor-joining (NJ) method. Numbers at nodes indicate bootstrap values (1,000 replicates) for the maximum parsimony, maximum likelihood, and NJ methods. Plerocercoid 1 was from Dinodon ruforzonatum ruforzonatum in Korea, Plerocercoid 2 was from Dinodon ruforzonatum ruforzonatum in China, Plerocercoid 3 was from Rhabdophis tigrinus tigrinus in Korea, Plerocercoid 4 was from Agkistrodon saxatilis in Korea, and Plerocercoid 5 was from Agkistrodon saxatilis in China. 


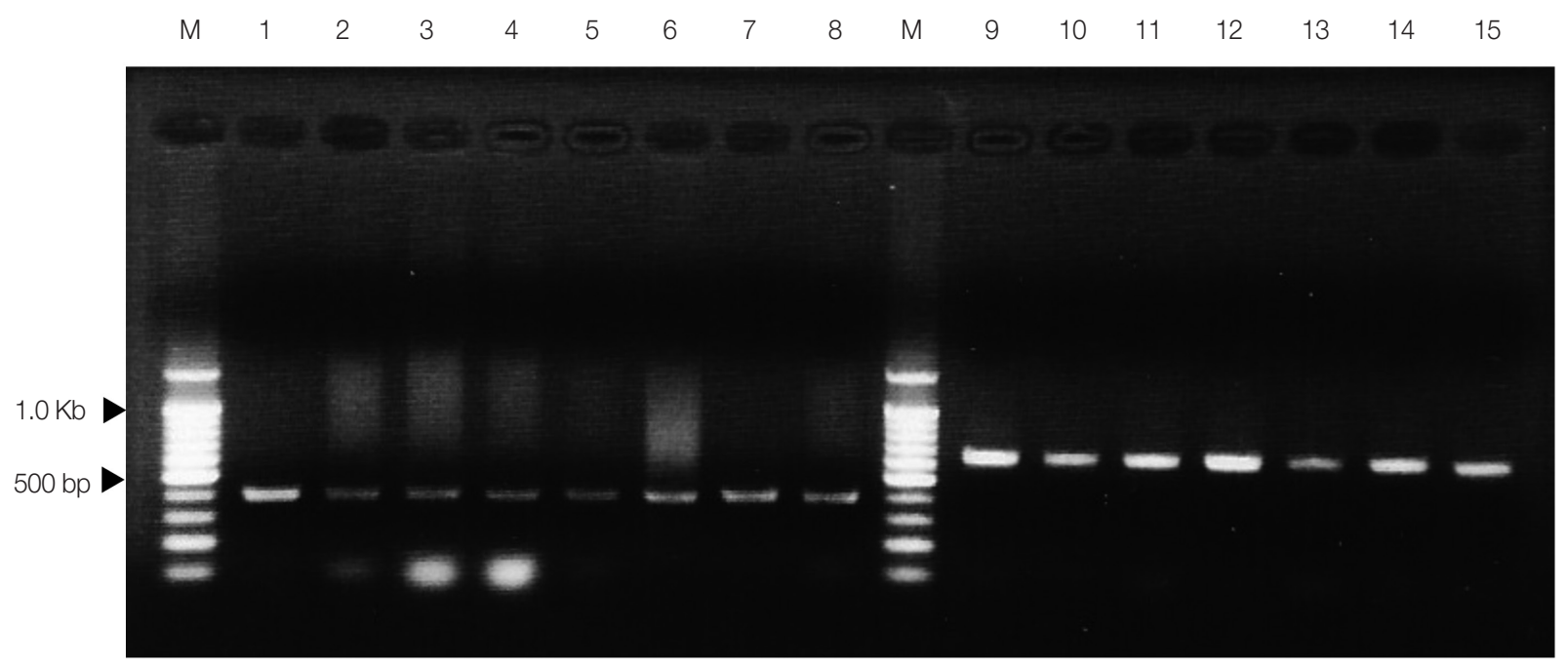

Fig. 2. Multiplex PCR with a mixture of all primers and genomic DNA from S. erinaceieuropaei and $S$. decipiens used in the present study. Lane M, DNA size maker (100 bp ladder); lanes 1-8: S. erinaceieuropaei (401 bp); lanes 9-15: S. decipiens (644 bp).

ods (MP, NJ, and ML) shown in Fig. 1. In the present study, all of the 50 spargana randomly chosen and comprised of 10 specimens from each of 5 species of snakes that included $D$. rufozonatum rufozonatum (Korean and Chinese), R. tigrinus tigrinus (Korean), and A. saxatilis (Korean and Chinese) were identified as $S$. decipiens using nucleotide sequence analysis of mitocondrial cox1.

\section{Differential diagnosis of spirometrid tapeworms by multiplex PCR}

The results of PCR amplification of spirometrid tapeworm DNA using multiplex PCR are presented in Fig. 2. The PCR products obtained using the species-specific primers were positively detected in all PCR tested with a mixture of S. erinaceieuropaei and S. decipiens DNA templates. The S. erinaceieuropaeispecific bands (401 bp) were obtained in PCR with a mixture of S. erinaceieuropaei-specific primers (Se/Sd-7963F, forward; Se-8344bands, reverse) and S. erinaceieuropaei and S. decipiens DNA templates (lanes 1-8 in Fig. 2). S. decipiens-specific bands (644 bp) were also detected in PCR with a mixture of S. decipiens-specific primers (Se/Sd-7963F, forward; Sd-8584R, reverse) and $S$. decipiens and S. erinaceieuropaei DNA templates (lanes 9-15 in Fig. 2). These PCR products were cloned and sequenced to confirm the specificity of each species-specific primer. The results of sequence analyses for the species-specific bands revealed a $100 \%$ sequence identity with homologous regions of the mtDNA sequences of $S$. erinaceieuropaei and $S$. decipiens. In order to identify spargana species, multiplex PCR was applied to the remaining 854 spargana. The species-specific 644 -bp diagnostic band was detected in all of the 854 spargana subjected to multiplex PCR (data not shown).

\section{DISCUSSION}

It is known that the majority of human sparganosis cases are caused by consumption of raw reptiles (snakes), amphibians (frogs), or drinking water contaminated with larval Spirometra species. Regarding the well-known species Spirometra erinaceieuropaei, Cho et al. [2] investigated 56 cases in search of possible sources of infections for human sparganosis. Approximately a half of the cases $(n=30)$ were involved with consumption of raw snakes, and 10 cases were associated with drinking untreated water. Thus, the consumption of raw snake flesh has been considered the most important cause of human sparganosis in Korea. This was generally accepted by most parasitologists without opposition; however, our research group recently suggested that $S$. erinaceieuropaei is not the only species that induces human sparganosis [4]. The suggestion was that $S$. decipiens is another possible cause of human sparganosis in Korea. The species ratio presented in the report was 35 (S. erinaceieuropaei): 15 (S. decipiens) for cases of human sparganosis among Korean patients recorded for the period 19792009 from all of the parasitology laboratories in Korea. The simplified ratio of 2.3:1 reveals approximately twice as many cases of S. erinaceieuropaei than $S$. decipiens among the 50 specimens examined [4]. This finding immediately provoked ques- 
tions concerning whether S. erinaceieuropaei and S. decipiens are caused by the same source of infection, namely, snakes, and if so, what is the ratio between these Spirometra species in the snakes? To answer these questions, genetic analyses of spargana that infect snakes are required to identify the Spirometra species. Our present study revealed a rather surprising result that all spargana from snakes were identified as $S$. decipiens $(\mathrm{n}=904)$ without exception. This finding highlights a serious epidemiological discrepancy between the ratios of Spirometra species in humans and snakes.

We do not currently know the reason why we did not find any S. erinaceieuropaei from any of our snakes. One possible explanation is that the S. erinaceieuropaei larval stage may be the primary source of human infection by way of drinking water contaminated with procercoids, but this then begs an even bigger question: what is the intermediate host that transfers larval forms to humans if it is not snakes? Furthermore, what is the final host? However, all these questions are based on a supposition that there are 2 different kinds of Spirometra in humans. The use of a poultice, which represents another form of spargana transmission to humans, was not taken seriously because it seems that it is not commonly practiced nowadays. The final hosts of Spirometra spp. are commonly known to be carnivorous animals such as cats and dogs, but $S$. erinaceieuropaei has not been found or ever described in natural infections in cats and dogs [9]. Thus, S. decipiens might be the only species of naturally occurring tapeworm that is currently found in cats and dogs. The only clearly known second intermediate host of S. erinaceieuropaei is the Chinese hedgehog. These findings indicate that further studies are required to determine the real infection route and final hosts of S. erinaceieuropaei, and to clarify whether $S$. decipiens infects humans through drinking water contaminated with procercoids.

In the present study, the differential diagnosis obtained by PCR using species-specific primers was correlated with species identification based on nucleotide sequence analysis of the mitochondrial cox1 gene. The results showed that the multiplex PCR assay with the Se/Sd-7963F, Se-8344R, and Sd8584R primers will be useful for species identification in the genus Spirometra. Finally, the spargana that infected snakes ( $D$. rufozonatum rufozonatum, R. tigrinus tigrinus, and A. saxatilis) in Korea and China was $S$. decipiens, and this species was distinguished from the spargana of S. erinaceieuropaei using analysis of mitochondrial cox1 sequences and multiplex PCR. We wonder if our mysterious finding that S. erinaceieuropaei larval forms were not identified in snake specimens collected from Korea and China will be explained in the near future if investigations use larger sample sizes.

\section{ACKNOWLEDGMENTS}

The National Research Foundation of Korea (NRF-2012 R1A1A2042993) supported one of the authors (Hyeong-Kyu Jeon) with a Research Fellow Grant for young scientists. This work was also supported by a research grant from Chungbuk National University in 2013.

\section{CONFLICT OF INTEREST}

We have no conflict of interest related to this work.

\section{REFERENCES}

1. Uemura S. On the Ligula mansoni from human. J Chosen Med Ass 1917; 20: 114 (in Japanese).

2. Cho SY, Bae JH, Seo BS. Some aspects of human sparganosis in Korea. Korean J Parasitol 1975; 13: 60-77.

3. Min DY. Cestode infections in Korea. Korean J Parasitol 1990; 28 (suppl): 123-144.

4. Jeon HK, Park HS, Lee DM, Choe SJ, Kim KH, Huh S, Sohn WM, Chai JY, Eom KS. Human infections with Spirometra decipiens plerocercoids identified by morphologic and genetic analyses in Korea. Korean J Parasitol 2015; 53: 299-305.

5. Kobayashi H. On the animal parasites in Korea. Japan Med World 1925; 5: 9-16 (in Japanese).

6. Weinstein PP, Krawczyk HJ, Peers JH. Sparganosis in Korea. Am J Trop Med Hyg 1954; 3: 112-129.

7. Seo BS, Cho SY. Endoparasites of snakes from Keo-je Island. Korean J Parasitol 1964; 2: 130-131 (in Korean).

8. Cho SY, Hwang KI, Seo BS. On the Sparganum mansoni infection in some Korean terrestrial snakes. Korean J Parasitol 1973; 11: 87-94.

9. Faust EC, Campbell HE, Kellogg CR. Morphological and biological studies on the species of Diphyllobothrium in China. Am J Hyg 1929; 9: 560-583.

10. Eom KS, Park HS, Lee DM, Choe SJ, Kim KH, Jeon HK. Mitochondrial genome sequences of Spirometra erinaceieuropaei and $S$. decipiens (Cestoidea: Diphyllobothriidae). Korean J Parasitol 2015; 53: 455-463.

11. Swofford DL. Paup*: phylogenetic analysis using parsimony (*and other methods). Version 4. Sunderland, Massachusettes, USA. Sinauer Associates. 2003. 